\title{
Clinical and economic aspects of sevelamer therapy in end-stage renal disease patients
}

This article was published in the following Dove Press journal: International Journal of Nephrology and Renovascular Disease 8 May 2014

Number of times this article has been viewed

\section{Shahrzad Ossareh}

Department of Medicine, Nephrology Section, Hasheminejad Kidney Center, Iran University of Medical Sciences, Tehran, Iran
Correspondence: Shahrzad Ossareh Hemodialysis Ward, Hasheminejad Kidney Center, Iran University of Medical Sciences, Tehran, Iran Tel +982 2I 88644420

Fax +98 2I 8864444 I

Email ossareh_s@hotmail.com
Abstract: Phosphate control is still a great challenge in chronic kidney disease (CKD), and in spite of the great improvements in dialysis techniques, achievement of the goals for mineral metabolism control is still far from ideal. Aluminum hydroxide has been largely abandoned due to the high risk of aluminum toxicity, while the use of calcium-based phosphate binders may cause hypercalcemia, overzealous parathyroid suppression, and extraskeletal calcification. Sevelamer hydrochloride has been introduced as an efficient medication for phosphate control, with a lower risk of hypercalcemia and parathyroid suppression. Various clinical trials have compared the risk of vascular calcification between sevelamer and calcium salts with inconsistent results. In spite of these inconsistencies, the Kidney Disease Outcomes Quality Initiative (KDOQI) suggests non-calcium phosphate binders as the preferred phosphate binder in dialysis patients with severe vascular and/or other soft-tissue calcifications and in those with hypercalcemia or parathyroid hormone $(\mathrm{PTH})<150 \mathrm{mg} / \mathrm{dL}$. The Kidney Disease Improving Global Outcome (KDIGO) limits the use of non-calcium phosphate binders to patients with hypercalcemia. Regarding the effect on mortality, the results of clinical trials are again inconsistent. The other important aspect of using sevelamer is the issue of price, which is substantially higher than calcium-based phosphate binders. Reviewing the studies on economic aspects shows that sevelamer increases quality-adjusted life-years (QALY) and possibly life years, with a higher cost compared to calcium-based phosphate binders. In conclusion, sevelamer is a very useful drug for phosphate control, reduction of hypercalcemia, and lessening the risk of adynamic bone disease, with probable reduction in vascular calcification and possible reduction in mortality rate. It has a higher economic burden on health care systems compared to calcium-based phosphate binders. This may affect its extensive use according to guideline recommendations, and will be influenced by local health care budgets and the decision of health care strategists.

Keywords: sevelamer, phosphate binder, vascular calcification, hyperphosphatemia, QALY per cost

\section{Introduction}

Great improvements have been made in the management of end-stage renal disease (ESRD) during the last few decades, including better modalities of dialysis with modern dialysis machines and high quality dialyzers. However phosphate control is still a major challenge in this group of patients, and achievement of the goals provided by guidelines has not been better than $53 \%-75 \%$ in patients on chronic hemodialysis or peritoneal dialysis in different reports. ${ }^{1,2}$ Phosphorus retention, which starts early in chronic kidney disease (CKD), is considered to be one of the main cardiovascular risk factors for patients with CKD. ${ }^{3}$ In these stages, phosphate retention leads to increased secretion of parathyroid hormone (PTH) and fibroblast growth factor-23 (FGF-23), which help to maintain 
normophosphatemia via increased urinary phosphate excretion. FGF-23 also decreases 1,25 dihydroxy-D3 production and suppresses intestinal phosphate absorption. ${ }^{4}$ However these mechanisms gradually become less efficient in maintaining normal phosphate levels, which is at least partially due to resistance of the kidneys and parathyroid glands to inhibit the effect of FGF-23 on phosphate excretion and PTH secretion in the context of Klotho and FGF receptor 1 downregulation in these organs. ${ }^{5,6}$ Therefore, in the later stages of CKD, increased phosphate level, together with higher levels of calcium-phosphate products, PTH, and FGF-23 levels, occur almost inevitably. Increased phosphate levels have been attributed to the development of vascular calcification in CKD patients. ${ }^{7}$ Higher serum phosphorus levels, even in the normal range, are correlated with aortic valve sclerosis and aortic and mitral annular calcification in the elderly population. ${ }^{8,9}$

Due to these considerations, the 2009 Kidney Disease: Improving Global Outcomes (KDIGO) clinical practice guidelines recommend to keep serum phosphorus levels in the normal range in patients with CKD stages 3-5 and toward normal in dialysis patients. ${ }^{10}$ By comparison, the National Kidney Foundation Kidney Disease Outcomes Quality Initiative (NFK-K/DOQI) recommends that serum levels of phosphate should be maintained between 3.5 and $5.5 \mathrm{mg} / \mathrm{dL}$, which is a more specific goal, assumed to be the inflection point above which patient survival significantly deteriorates. ${ }^{4,11}$

To achieve these goals, dietary phosphate restriction, together with the use of phosphate binders may be started in the relatively early stages of CKD and is actually implied in almost all patients in CKD stages 4 and 5. Restricting phosphate-containing foods, especially food additives and fast foods, while keeping high biologic value proteins such as meat and egg in the daily diet, can significantly decrease serum phosphate level. ${ }^{12}$ Even careful attention to food labels describing the food additives, is effective in improving hyperphosphatemia. ${ }^{13}$ However, dietary phosphorus restriction is usually not limited to food additives and usually major protein restrictions are recommended to patients. This may lead to protein malnutrition and decreased normalized protein equivalent of total nitrogen appearance (nPNA), and outweigh the benefits of controlled phosphorus, finally leading to greater mortality. ${ }^{14}$ Therefore, phosphate control generally needs a (number of) phosphate binder(s), especially in patients in the later stages of CKD and those on dialysis.

\section{Phosphate binders}

To date, there have been many studies linking high serum phosphate level to mortality; however, there are no randomized studies showing the beneficial effect of the administration of phosphate binders on patient survival. There is only one large 1-year prospective observational study on 10,044 incident hemodialysis patients, which showed a significant survival advantage with using phosphate binders compared with no treatment. ${ }^{15}$ However, due to the large pool of data showing the adverse effects of high phosphorus levels on skeletal and cardiovascular morbidity of CKD patients, phosphate binders are extensively used in these patients.

Phosphate binders are traditionally divided into calciumcontaining (calcium carbonate, calcium acetate) and noncalcium-containing (sevelamer, lanthanum carbonate, and rarely aluminum hydroxide and magnesium hydroxide) types. Aluminum hydroxide has been largely abandoned due to fear of aluminum toxicity with resultant osteomalacia, dementia, myopathy, and anemia, though in severe (serum phosphorus $>7 \mathrm{mg} / \mathrm{dL}$ ) and refractory hyperphosphatemia, short courses of aluminum hydroxide (up to 4 weeks, one time only) have been recommended, to be replaced by another phosphate binder afterwards. ${ }^{16,17}$ Magnesium hydroxide is also generally avoided due to the risk of hypermagnesemia and frequent occurrence of diarrhea.

Calcium-containing phosphate binders are cheap and effective, and provide an important mineral, ie, calcium. They can maintain a positive calcium balance along with their phosphate-binding effect. A risk of hypercalcemia in up to $50 \%$ has been reported with these agents, although it seems that such a high risk has been due to the high doses of calcium salts used in initial reports, together with high dialysate calcium baths. ${ }^{18,19}$ On the other hand, there is a risk of oversuppression of parathyroid hormone (PTH), adynamic bone disease, and vascular calcification with these agents, which cannot be overlooked. ${ }^{20,21}$ Hence the latest KDIGO guidelines recommend to restrict the dose of calcium-based phosphate binders in the presence of persistent or recurrent hypercalcemia and suggest the same restriction in the presence of arterial calcification, adynamic bone disease, or persistent low serum PTH. ${ }^{22}$

\section{Sevelamer}

Sevelamer hydrochloride (Renagel; Sanofi, Paris, France) was the first synthetic non-aluminum, non-calcium, and non-absorbable phosphate binder introduced and tested on normal volunteers as well as dialysis patients in $1997 . .^{23-25}$ The primary pharmacologic effect of sevelamer was binding to bile acids, increasing fecal bile excretion, and LDLcholesterol lowering. ${ }^{24}$ Afterwards, in several open-label studies, both on patients on dialysis or with renal failure, 
sevelamer was showed to be as effective as calcium-based binders in lowering phosphate, but without the risk of hypercalcemia. ${ }^{26-30} \mathrm{~A}$ decrease in serum LDL was also shown as a beneficial co-effect. ${ }^{27,31}$ Sevelamer carbonate (Renvela; Sanofi) was introduced and approved by US Food and Drug administration for control of hyperphosphatemia in dialysis patients years later, ie, in 2007.

Different issues should be discussed regarding sevelamer, including its effects on vascular calcification, bone disease and mortality, biochemical effects, and expenses. In the following sections, we will address these issues according to the existing evidence.

\section{Arterial calcification, hypercalcemia, PTH level}

Although the data are still inconsistent, there appears to be less progression of arterial calcification with sevelamer compared to calcium-based phosphate binders. In the "Treat-to-goal" (TTG) randomized clinical trial, Chertow et al showed a lower likelihood of hypercalcemia, lower levels of PTH, and less progressive coronary and aortic calcification in 99 hemodialysis patients on sevelamer compared to 101 patients on calcium salts. ${ }^{32}$ Routine biochemical parameters were the same in both groups, except for a lower level of bicarbonate in patients on sevelamer. However, at the end of this study, patients on sevelamer had lower total and LDL cholesterol, and the question remained whether the lower calcium burden or the lower LDL cholesterol were protective for the progression of coronary artery calcification (CAC). In another study, the same group showed that in patients on calcium-based phosphate binders, progressive coronary artery and aortic calcification, as measured by electron beam tomography, were associated with higher time-averaged concentrations of calcium, phosphorus, and the calcium-phosphorus product on the one hand and lower PTH on the other. ${ }^{33}$ Such a relationship was not found in patients on sevelamer. In the "Renagel in New Dialysis" (RIND) trial on new hemodialysis patients, those with evidence of at least mild coronary calcification had significant progression in calcification score at 6,12 , and 18 months. ${ }^{34}$ Use of calcium-containing phosphate binders resulted in more rapid progression of CAC than did use of sevelamer hydrochloride. Subjects treated with sevelamer had significantly lower mean corrected calcium, higher PTH, and lower total and LDL cholesterol. In a recent randomized trial on two parallel groups of hemodialysis patients, there was a significantly smaller increase in the progression of the CAC score in the sevelamer group compared to the calcium carbonate group after 6 months of follow-up. ${ }^{35}$ Interestingly, an increase in advanced glycation end products, as represented by pentosidine, was seen in patients treated by calcium carbonate but not by sevelamer.

There are other studies, however, such as the Calcium Acetate Renagel Evaluation 2 (CARE-2) study, which do not support the superiority of sevelamer in terms of the prevention of vascular calcification. ${ }^{36}$ In this study, 200 hemodialysis patients were randomized to either sevelamer or calcium acetate for 1 year. Atorvastatin was added to achieve serum LDL cholesterol levels $<70 \mathrm{mg} / \mathrm{dL}$ in both groups. At 1 year, patients treated with either calcium acetate or sevelamer experienced similar progression of CAC. There were a number of limitations in this study, however, including the non-inferiority trial design with a wide a priori margin of 1.8, a significant difference in end-of-study PTH levels between the two groups, and large number of dropouts. ${ }^{36,37}$ Another study from Brazil (the Phosphate Binder Impact on Bone Remodeling and Coronary Calcification or BRiC study) also did not show any difference between vascular calcification score in dialysis patients receiving either calcium acetate or sevelamer. ${ }^{38}$ In this study, in spite of similar dialysate calcium concentrations between the two groups at the beginning of the study, patients on sevelamer were treated with higher dialysate calcium during the trial. This may have mitigated any differences in vascular calcification score between the treatment groups. ${ }^{37}$

In a systematic review of phosphate binders in CKD which examined 40 trials with 6,406 patients, there was a significant decrease in end-of-treatment phosphorus and PTH levels with calcium salts when compared with sevelamer and a significant decrease in the risk of hypercalcemia (pooled data, relative risk [RR]: 0.47; 95\% confidence interval [CI]: 0.36-0.62) with sevelamer compared with calcium-based agents. ${ }^{39}$ The findings of four trials which were examined in this systematic review in terms of vascular calcification could not be pooled due to different scoring systems for the assessment of vascular calcification, and had quite diverse results, with decreased risk of vascular calcification in patients using sevelamer in two trials and similar risk between sevelamer and calcium salts in the other two.

There are also a number of meta-analyses examining the difference in the progression of vascular calcification between sevelamer and calcium-containing phosphate binders, with conflicting results. Two recent meta-analyses by Jamal et al, including five trials on CAC, and Zhang et al, including 14 studies, do not find a significant difference in CAC score between the patients on sevelamer or calcium-containing phosphate binders. ${ }^{40,41}$ 
Overall, the results are still inconsistent and the differences in study design and population, dialysate calcium concentration, PTH control targets, and use of vitamin D (analogs) may all have affected the findings. Furthermore, according to statistical probability, when several small clinical trials are performed, the null hypothesis will not be rejected in some cases even when true differences exist. ${ }^{37}$

In spite of the inconsistencies between the results of clinical trials and meta-analyses, the National Kidney Foundation Kidney Disease Outcomes Quality Initiative (NKF-KDOQI) has provided recommendations which may help in the clinical management of hyperphosphatemia in CKD patients. They state that both calcium-based- and noncalcium-, non-aluminum-, and non-magnesium-containing phosphate binders are effective in lowering serum phosphorus levels and may either be used as the primary therapy, or in combination. ${ }^{11}$ The total dose of elemental calcium provided by the calcium-based phosphate binders should not exceed 1,500 mg/day and calcium-based phosphate binders should not be used in dialysis patients who are hypercalcemic or whose plasma PTH levels are $<150 \mathrm{pg} / \mathrm{mL}$. Non-calcium phosphate binders are preferred in dialysis patients with severe vascular and/or other soft-tissue calcifications. On the other hand, the Kidney Disease Improving Global Outcome (KDIGO) group believes that in the absence of hypercalcemia, there is no indication to prescribe phosphate binders that are less cost-effective than calcium-based agents. ${ }^{10}$

\section{Mortality}

There are again conflicting results regarding the effect of sevelamer on mortality, either alone or when compared to calcium salts.

In a post-hoc analysis of the RIND study, at a median follow-up of 44 months, mortality was significantly lower in patients on sevelamer compared to those on calcium salts (5.3/100 versus $10.6 / 100$ patient years, $P=0.05)$ which persisted after multivariable adjustment $(P=0.016$, hazard ratio [HR]: 3.1$){ }^{42}$ In addition, in subjects new to hemodialysis, the baseline CAC score was a significant predictor of allcause mortality, and treatment with sevelamer was associated with a significant survival benefit as compared to the use of calcium-containing phosphate binders.

In a recent study, $212 \mathrm{CKD}$ stage 3-4 patients were nonblindly randomized to either sevelamer or calcium carbonate and the patients were followed for 36 months. ${ }^{43}$ In patients randomized to sevelamer, all-cause mortality and the composite endpoint were lower but there were no advantages in dialysis inception.
The DCOR (Dialysis Clinical Outcomes Revisited) trial was a randomized, open-label, parallel design and an effectiveness trial, which compared all-cause and causespecific mortality among hemodialysis patients treated with calcium-based phosphate binders or sevelamer, in a prevalent dialysis population. ${ }^{44} \mathrm{~A}$ total of 2,103 patients were initially randomized to treatment and 1,068 patients completed the study. All-cause mortality rates and cause-specific mortality rates were not significantly different between the two groups. Only in patients $>65$ years of age was there a significant effect of sevelamer in lowering the all-cause but not cardiovascular mortality rate. The authors suggested that their findings in older subjects could be because older hemodialysis patients tend to have greater calcification burden than younger patients, and therefore, calcium could affect them over a shorter follow-up period. They explained the lack of difference in cardiovascular mortality between the two groups by the fact that the study was not powered to detect differences on cardiovascular or other causes of mortality in design. A secondary analysis of the results of the DCOR study showed that compared with calcium salts, treatment with sevelamer did not affect overall or cause-specific mortality, morbidity, or first or cause-specific hospitalization, but that there was evidence for a beneficial effect on multiple all-cause hospitalizations and hospital days..$^{45} \mathrm{~A}$ systematic review on clinical efficacy and safety of sevelamer in dialysis patients showed that compared with calcium-based phosphate binders, sevelamer was associated with similar to slightly higher levels of phosphate, similar calcium-phosphate product levels, and slightly lower serum calcium levels. ${ }^{46}$ There was no evidence that sevelamer reduced all-cause mortality, cardiovascular mortality, the frequency of symptomatic bone disease, or health-related quality of life (QOL).

Overall, it seems that in spite of more than 15 years of experience with sevelamer and decades of experience with calcium-based phosphate binders, there is still room for more studies on targeted endpoints and achievement of more clear-cut results before we can achieve any consensus on the different aspects of the clinical efficacy of these drugs.

\section{Safety issue: metabolic acidosis}

A number of adverse effects have been associated with sevelamer, especially metabolic acidosis. A study on 16 hemodialysis patients was performed with 2 weeks of washout from calcium carbonate and 6 weeks of subsequent sevelamer therapy. ${ }^{47}$ At the end of the washout period, the serum bicarbonate level decreased from $20.1 \pm 2.6$ to $18.2 \pm 3.1 \mathrm{mmol} / \mathrm{L}$ and further to $17.3 \pm 3.2 \mathrm{mmol} / \mathrm{L}$ in the first week of treatment with 
sevelamer $\mathrm{HCl}$. Bicarbonate levels remained stable during the following weeks of treatment, and during the 2-week washout after discontinuation of sevelamer. They concluded that reduction of serum bicarbonate was dependent on the withdrawal of calcium carbonate and the consequent reduced intake of alkali, rather than on treatment with sevelamer $\mathrm{HCl}$. On the other hand, sevelamer is a non-absorbable polymer chain which contains $17 \%$ chloride by weight, as amine hydrochloride. Brezina et al believe that it may inherently cause metabolic acidosis by three potential mechanisms. ${ }^{48}$ The first suggested mechanism is release of one molecule of hydrochloric acid from the amine resin for each molecule of phosphate bound in the gut and subsequent production of $\mathrm{HCl}$. The second mechanism is sequestration of bile acids by sevelamer in exchange for the release of chloride, and again resultant production of one $\mathrm{HCl}$ molecule for every molecule of bile acid bound. Also, there is a local concentration of $100-120 \mathrm{mEq} / \mathrm{L}$ of bicarbonate in the small intestine from pancreatic secretions which may bind to sevelamer in exchange for chloride and lead to the formation of loose stools containing carbonated sevelamer with ensuing metabolic acidosis similar to chronic diarrhea. A number of other studies have confirmed the risk of metabolic acidosis with sevelamer. ${ }^{31,49,50}$ To overcome this risk in the already acidotic dialysis patients and its subsequent adverse effects on bone, reformulation of sevelamer hydrochloride was suggested and led to the production of sevelamer carbonate (Renvela). ${ }^{51}$ This drug has been shown to be equivalent in controlling serum phosphorus and lipids in hemodialysis patients with increased serum bicarbonate levels when compared to Renagel. ${ }^{52}$ It should be mentioned that there are some concerns about the name of sevelamer "carbonate", as the resin dissociates into free sevelamer and sevelamer "bicarbonate" in the acidic $\mathrm{pH}$ of gastrointestinal tract, which is well below the isoelectric $\mathrm{pH}$ of sevelamer "carbonate", 53

The other side effect of sevelamer is increased risk of gastrointestinal upset, including abdominal bloating, diarrhea, and constipation compared with calcium salts (RR: 1.39). ${ }^{39}$

\section{Effect on bone}

There does not seem to be a major difference between sevelamer and calcium-based phosphate binders in terms of bone histology, although the results of the studies are not consistent. Barreto et al randomized 101 hemodialysis patients with similar baseline characteristics to either to sevelamer or calcium acetate. ${ }^{38}$ After 1 year, the 71 patients who concluded the study had similar levels of calcium and phosphorus, with a higher PTH, bone alkaline phosphatase and deoxyproline, and lower LDL cholesterol in the sevelamer group. Progression of CAC and bone histological diagnosis at baseline and 12 months were similar in both groups. Patients with baseline low bone turnover disease had an improvement in bone formation rate on either drug; however, those with baseline high bone turnover disease had an increase in bone resorption if on sevelamer. The authors concluded that there was no difference in CAC progression or changes in bone remodeling between the calcium and the sevelamer groups; however, the finding of increased bone resorption with sevelamer in high bone turnover disease needs special consideration and studies with larger sample sizes.

In another randomized study on 119 hemodialysis patients with adynamic bone disease as the most common baseline bone histology, there were no changes in mineralization lag time or measures of bone turnover after 1 year in either the calcium carbonate or sevelamer groups. ${ }^{54}$ Osteoid thickness significantly increased in both groups, with no significant difference between them. Bone formation rate per bone surface, however, significantly increased from baseline only in the sevelamer group $(P=0.019)$ and trabecular architecture improved with sevelamer in those with abnormal trabecular structure. The latter findings raise the possibility that sevelamer may increase bone formation rate during the treatment of adynamic bone disease, an effect that might be more obvious in a larger, more homogeneous sample size. ${ }^{37}$

Comparing the results of these studies, can we conclude that sevelamer may increase bone resorption in high bone turnover disease and increase bone formation rate in low bone turnover disease? Can we make a choice of the type of phosphate-binding agent according to bone histology?

In a trial on 29 pediatric patients with secondary hyperparathyroidism $\left(2^{\circ} \mathrm{HPT}\right)$ and osteitis fibrosa, both sevelamer and calcium carbonate improved the skeletal lesions of $2^{\circ} \mathrm{HPT}$, with similar control of serum phosphorus, lower calcium concentration, and higher PTH level in the sevelamer group. ${ }^{55}$ However, in patients on sevelamer, the dose of active vitamin D sterols was increased during the study and reduction in PTH and alkaline phosphatase levels occurred late, largely during the last 4 months of the study. ${ }^{55}$ This shows that the children treated with sevelamer may have needed a higher dose of active vitamin D sterols for the control of $2^{\circ} \mathrm{HPT}$. The group of children on sevelamer ingested a smaller than recommended dose of calcium, suggesting that, at least in children, insufficient calcium intake may exacerbate the mineralization defect. ${ }^{37}$

Overall, it seems that we still need further randomized trials, especially based on bone histology, to give more definite answers to the above questions. 


\section{Economic aspects}

Apart from clinical issues, an important concern in the regular use of sevelamer as a substitution for calcium-based phosphate binders is the cost of the medication and the economic burden on health care systems. The cost of each $800 \mathrm{mg}$ tablet of Renagel is about US\$4.79 and Renvela US\$3.83, compared to US $\$ 0.84$ for $667 \mathrm{mg}$ calcium acetate (PhosLo; Fresinius Medical Care North America, Waltham, MA, USA) and US\$0.06 for $750 \mathrm{mg}$ calcium carbonate (Tums; GlaxoSmithKline, St Louis, MO, USA). ${ }^{56}$ In many countries, sevelamer is not under insurance coverage and it may actually be impossible for the majority of patients to get the drug.

Clearly, sevelamer is much more expensive than calciumcontaining phosphate binders. However, if it can reduce medical services including hospitalization costs, this higher price may be worth it. There are a number of studies examining the different economic aspects of sevelamer.

A systematic review of sevelamer in ESRD, which analyzed its potential economic impact in Canada and the US in 2004 , showed that at least $51 \%$ and $64 \%$ of dialysis patients in the Canadian and US cohorts, respectively, would meet K/ DOQI criteria for use of sevelamer and that this would require approximately US\$26 and US\$780 million per year in these two countries. ${ }^{57}$ They concluded that given the potential budgetary impact, future nephrology clinical practice guidelines should consider resource use, in addition to clinical data. The same group did another study examining the quality-adjusted life-years (QALY) per cost, based on data of patients from the Canadian Organ Replacement Registry (CORR), the United States Renal Data System (USRDS), and the DCOR study. ${ }^{58}$ Their study showed that the cost per QALY gained for treating all dialysis patients with sevelamer exceeded the acceptable amount, even after excluding the costs of dialysis and transplant from the calculations. For patients $>65$ years, considering the survival benefit gained by sevelamer in the DCOR study, the strategy remained economically unattractive..$^{58}$ Pointing to a trend towards a reduction in hospitalization rates (which would generate cost savings), in the DCOR study, they concluded that assuming no difference in survival, the risk of hospitalization would have to be lowered by $30 \%$ to offset the additional medication cost of sevelamer. ${ }^{58}$ As in this study many cost inputs were estimated due to unavailability of actual costs, another study was performed as a secondary economic analysis of the DCOR trial, in which Medicare costs for most of the study participants could be directly evaluated. ${ }^{59}$ They evaluated the Medicare total, inpatient, outpatient, skilled nursing facility, and other costs for sevelamer- versus calcium-treated patients, in addition to the costs of the medications themselves. The results of DCOR had shown no difference in overall mortality, but suggested a significantly lower hospitalization rate and number of hospital days for patients using sevelamer versus calcium carbonate. ${ }^{44}$ Thus, in this study, the authors intended to understand whether the increased cost of therapy is offset by decreased medical costs. ${ }^{59}$ They showed that Medicare costs were US $\$ 2,388$ less for sevelamer-treated than for calcium-treated patients, mainly due to lower hospitalization costs. Outpatient costs were similar for the sevelamer and calcium groups despite outpatient monthly intravenous vitamin D costs of US $\$ 82$ per patient higher for sevelamer-treated than for calcium-treated patients. However, once the costs of the phosphate binders were included, overall monthly medical costs were greater for sevelamer-treated than for calcium-treated patients by US $\$ 81$ per patient using average wholesale price and US\$25 per patient, using wholesale acquisition costs..$^{59}$

In another study, Thompson et al in the UK used data from the INDEPENDENT-CKD study, to estimate total life years (LYs), QALYs, and costs, for patients treated with sevelamer or calcium carbonate. ${ }^{43,60}$ Incremental costs per LY gained (LYG) and cost per QALY gained for sevelamer versus calcium carbonate, were evaluated, using a Markov decision analytic model. Over a lifetime horizon, sevelamer treatment resulted in a gain of 2.05 LYs and 1.56 QALYs per patient, an increase of $£ 37,282$ in total costs per patient versus calcium carbonate, and a per-patient cost of $£ 18,193$ / LYG and $£ 23,878$ /QALY gained. The probabilistic sensitivity analysis showed that sevelamer was cost-effective compared to calcium carbonate in $93 \%$ of simulations, at a willingnessto-pay threshold of $£ 30,000 / \mathrm{Q} A L Y$ gained. ${ }^{60}$ It should be noted that the patient cohort on INDEPENDENT- CKD study were in stage 3 and 4 of CKD and not dialysis patients, for whom the extra costs of dialysis should be included.

Another study that used the UK National Health Service (NHS) perspective and final results of the DCOR study, showed that the use of sevelamer resulted in a gain of $\sim 0.73$ LYs and 0.44 QALYs per patient. Total per-patient costs were higher for sevelamer, resulting in an incremental cost of $£ 22,157$ per QALY gained and £13,427 per LYG (in £2009). Increasingly favorable cost per QALY ratios were observed with increasing age. They concluded that in dialysis patients, treatment of hyperphosphatemia with sevelamer offers good value for money compared with calcium-based binders. ${ }^{61}$

Finally, Taylor et al found an incremental gain of 0.24 QALYs in CKD patients receiving sevelamer compared to calcium salts and almost similar cost per QALY with an incremental cost per QALY of $£ 27,120$ and an incremental 
cost per LYG of $£ 15,508$ for sevelamer, which was similar to calcium acetate. ${ }^{62}$ They concluded that together with the unique morbidity and mortality benefits, treatment with sevelamer confers clinical benefits with a modest investment of additional economic resources.

Overall, examining all of these studies, it seems that sevelamer increases QOL and possibly LYs, with a higher cost compared to calcium-based phosphate binders, and it will be a decision of health care strategists whether to accept these extra costs or not.

\section{Conclusion}

Sevelamer is a very useful drug for phosphate control, reduction of hypercalcemia, and lessening the risk of adynamic bone disease and vascular calcification, which could possibly lower mortality among CKD and dialysis patients. It has a higher economic burden on health care systems, which may partially be offset by a lower rate of hospitalization compared to calcium-based phosphate binders. It increases quality adjusted LYs, and although this may not be cost-beneficial in some societies, the great achievement of increased LYs and QOL cannot be undermined. Sevelamer is recommended in major CKD guidelines at least in special medical conditions. The situation may be totally different in developing countries with lower health care budgets and should be studied according to local socioeconomic and budget conditions.

\section{Disclosure}

The author reports no conflicts of interest in this work.

\section{References}

1. Wei M, Taskapan H, Esbaei K, Jassal SV, Bargman JM, Oreopoulos DG. $\mathrm{K} / \mathrm{DOQI}$ guideline requirements for calcium, phosphate, calcium phosphate product, and parathyroid hormone control in dialysis patients: can we achieve them? Int Urol Nephrol. 2006;38(3-4):739-743.

2. Craver L, Marco MP, Martínez I, et al. Mineral metabolism parameters throughout chronic kidney disease stages 1-5-achievement of K/DOQI target ranges. Nephrol Dial Transplant. 2007;22(4):1171-1176.

3. Kestenbaum B, Sampson JN, Rudser KD, et al. Serum phosphate levels and mortality risk among people with chronic kidney disease. $J \mathrm{Am} S o c$ Nephrol. 2005;16(2):520-528.

4. Martin KJ, González EA. Prevention and control of phosphate retention/ hyperphosphatemia in CKD-MBD: what is normal, when to start, and how to treat? Clin J Am Soc Nephrol. 2011;6(2):440-446.

5. Krajisnik T, Olauson H, Mirza MA, et al. Parathyroid Klotho and FGFreceptor 1 expression decline with renal function in hyperparathyroid patients with chronic kidney disease and kidney transplant recipients. Kidney Int. 2010;78(10):1024-1032.

6. Koh N, Fujimori T, Nishiguchi S, et al. Severely reduced production of klotho in human chronic renal failure kidney. Biochem Biophys Res Commun. 2001;280(4):1015-1020.

7. Ossareh S. Vascular calcification in chronic kidney disease: mechanisms and clinical implications. Iran J Kidney Dis. 2011;5(5):285-299.

8. Ossareh S. Cardiac valvular calcification in hemodialysis patients. Iran J Kidney Dis. 2013;7(2):77-79.
9. Linefsky JP, O’Brien KD, Katz R, et al. Association of serum phosphate levels with aortic valve sclerosis and annular calcification: the cardiovascular health study. J Am Coll Cardiol. 2011;58:291-297.

10. KDIGO 2012 Clinical Practice Guideline for the Evaluation and Management of Chronic Kidney Disease. Kidney Int Suppl. 2013; 3 Suppl:S136-S150.

11. K/DOQI clinical practice guidelines for bone metabolism and disease in chronic kidney disease. Am J Kidney Dis. 2003;42 Suppl 3:S1-S202.

12. Uribarri J, Calvo MS. Hidden sources of phosphorus in the typical American diet: does it matter in nephrology? Semin Dial. 2003;16(3): 186-188.

13. Sullivan C, Sayre SS, Leon JB, et al. Effect of food additives on hyperphosphatemia among patients with end-stage renal disease: a randomized controlled trial. JAMA. 2009;301(6):629-635.

14. Shinaberger CS, Greenland S, Kopple JD, et al. Is controlling phosphorus by decreasing dietary protein intake beneficial or harmful in persons with chronic kidney disease? Am J Clin Nutr. 2008;88(6):1511-1518.

15. Isakova T, Gutiérrez OM, Chang $\mathrm{Y}$, et al. Phosphorus binders and survival on hemodialysis. J Am Soc Nephrol. 2009;20(2):388-396.

16. Delmez JA, Slatopolsky E. Hyperphosphatemia: its consequences and treatment in patients with chronic renal disease. Am J Kidney Dis. 1992;19(4):303-317.

17. National Kidney Foundation. K/DOQI clinical practice guidelines for bone metabolism and disease in chronic kidney disease. Am J Kidney Dis. 2003;42 Supp1 3:S1-S201.

18. Schaefer K, Umlauf E, von Herrath D. Reduced risk of hypercalcemia for hemodialysis patients by administering calcitriol at night. Am J Kidney Dis. 1992;19(5):460-464.

19. Slatopolsky E, Weerts C, Lopez-Hilker S, et al. Calcium carbonate as a phosphate binder in patients with chronic renal failure undergoing dialysis. N Engl J Med. 1986;315(3):157-161.

20. Mohammed IA, Hutchison AJ. Phosphate binding therapy in dialysis patients: focus on lanthanum carbonate. Ther Clin Risk Manag. 2008;4(5):887-893.

21. Goodman WG, Goldin J, Kuizon BD, et al. Coronary-artery calcification in young adults with end-stage renal disease who are undergoing dialysis. N Engl J Med. 2000;342(20):1478-1483.

22. Chapter 4.1: KDIGO Clinical practice guideline for the diagnosis, evaluation, prevention, and treatment of Chronic Kidney Disease-Mineral and Bone Disorder (CKD-MBD). Kidney Int. 2009;76 Suppl 113: S50-S99.

23. Rosenbaum DP, Holmes-Farley SR, Mandeville WH, Pitruzzello M, Goldberg DI. Effect of RenaGel, a non-absorbable, cross-linked, polymeric phosphate binder, on urinary phosphorus excretion in rats. Nephrol Dial Transplant. 1997;12(5):961-964.

24. Burke SK, Slatopolsky EA, Goldberg DI. RenaGel, a novel calciumand aluminium-free phosphate binder, inhibits phosphate absorption in normal volunteers. Nephrol Dial Transplant. 1997;12(8):1640-1644.

25. Chertow GM, Burke SK, Lazarus JM, et al. Poly[allylamine hydrochloride] (RenaGel): a noncalcemic phosphate binder for the treatment of hyperphosphatemia in chronic renal failure. Am J Kidney Dis. 1997;29(1):66-71.

26. Bleyer AJ, Burke SK, Dillon M, et al. A comparison of the calcium-free phosphate binder sevelamer hydrochloride with calcium acetate in the treatment of hyperphosphatemia in hemodialysis patients. Am J Kidney Dis. 1999;33(4):694-701.

27. Goldberg DI, Dillon MA, Slatopolsky EA, et al. Effect of RenaGel, a non-absorbed, calcium- and aluminium-free phosphate binder, on serum phosphorus, calcium, and intact parathyroid hormone in end-stage renal disease patients. Nephrol Dial Transplant. 1998;13(9):2303-2310.

28. Slatopolsky EA, Burke SK, Dillon MA. RenaGel, a nonabsorbed calcium- and aluminum-free phosphate binder, lowers serum phosphorus and parathyroid hormone. The RenaGel Study Group. Kidney Int. 1999;55(1):299-307.

29. Ogata H, Koiwa F, Shishido K, Kinugasa E. Combination therapy with sevelamer hydrochloride and calcium carbonate in Japanese patients with long-term hemodialysis: alternative approach for optimal mineral management. Ther Apher Dial. 2005;9(1):11-15. 
30. Ketteler M, Rix M, Fan S, et al. Efficacy and tolerability of sevelamer carbonate in hyperphosphatemic patients who have chronic kidney disease and are not on dialysis. Clin J Am Soc Nephrol. 2008;3(4): $1125-1130$.

31. Sadek T, Mazouz H, Bahloul H, et al. Sevelamer hydrochloride with or without alphacalcidol or higher dialysate calcium vs calcium carbonate in dialysis patients: an open-label, randomized study. Nephrol Dial Transplant. 2003;18(3):582-588.

32. Chertow GM, Burke SK, Raggi P; Treat to Goal Working Group. Sevelamer attenuates the progression of coronary and aortic calcification in hemodialysis patients. Kidney Int. 2002;62(1):245-252.

33. Chertow GM, Raggi P, Chasan-Taber S, Bommer J, Holzer H, Burke SK. Determinants of progressive vascular calcification in haemodialysis patients. Nephrol Dial Transplant. 2004;19(6):1489-1496.

34. Block GA, Spiegel DM, Ehrlich J, et al. Effects of sevelamer and calcium on coronary artery calcification in patients new to hemodialysis. Kidney Int. 2005;68(4):1815-1824.

35. Kakuta T, Tanaka R, Hyodo T, et al. Effect of sevelamer and calcium-based phosphate binders on coronary artery calcification and accumulation of circulating advanced glycation end products in hemodialysis patients. Am J Kidney Dis. 2011;57(3):422-431.

36. Qunibi W, Moustafa M, Muenz LR, et al; CARE-2 Investigators. A 1-year randomized trial of calcium acetate versus sevelamer on progression of coronary artery calcification in hemodialysis patients with comparable lipid control: the Calcium Acetate Renagel Evaluation-2 (CARE-2) study. Am J Kidney Dis. 2008;51(6):952-965.

37. Raggi P, Vukicevic S, Moysés RM, Wesseling K, Spiegel DM. Ten-year experience with sevelamer and calcium salts as phosphate binders. Clin J Am Soc Nephrol. 2010;5 Suppl 1:S31-S40.

38. Barreto DV, Barreto Fde C, de Carvalho AB, et al. Phosphate binder impact on bone remodeling and coronary calcification-results from the BRiC study. Nephron Clin Pract. 2008;110(4):c273-c283.

39. Navaneethan SD, Palmer SC, Craig JC, Elder GJ, Strippoli GF. Benefits and harms of phosphate binders in CKD: a systematic review of randomized controlled trials. Am J Kidney Dis. 2009;54(4):619-637.

40. Jamal SA, Fitchett D, Lok CE, Mendelssohn DC, Tsuyuki RT. The effects of calcium-based versus non-calcium-based phosphate binders on mortality among patients with chronic kidney disease: a metaanalysis. Nephrol Dial Transplant. 2009;24(10):3168-3174.

41. Zhang Q, Li M, Lu Y, et al. Meta-analysis comparing sevelamer and calcium-based phosphate binders on cardiovascular calcification in hemodialysis patients. Nephron Clin Pract. 2010;115(4):c259-c267.

42. Block GA, Raggi P, Bellasi A, Kooienga L, Spiegel DM. Mortality effect of coronary calcification and phosphate binder choice in incident hemodialysis patients. Kidney Int. 2007;71(5):438-441.

43. Di Iorio B, Bellasi A, Russo D; INDEPENDENT Study Investigators. Mortality in kidney disease patients treated with phosphate binders: a randomized study. Clin J Am Soc Nephrol. 2012;7(3):487-493.

44. Suki WN; Dialysis Clinical Outcomes Revisited Investigators. Effects of sevelamer and calcium-based phosphate binders on mortality in hemodialysis patients: results of a randomized clinical trial. J Ren Nutr. 2008;18(1):91-98.

45. St Peter WL, Liu J, Weinhandl E, Fan Q. A comparison of sevelamer and calcium-based phosphate binders on mortality, hospitalization, and morbidity in hemodialysis: a secondary analysis of the Dialysis Clinical Outcomes Revisited (DCOR) randomized trial using claims data. Am J Kidney Dis. 2008;51(3):445-454.
46. Tonelli M, Wiebe N, Culleton B, et al; Alberta Kidney Disease Network. Systematic review of the clinical efficacy and safety of sevelamer in dialysis patients. Nephrol Dial Transplant. 2007;22(10):2856-2866.

47. Gallieni M, Cozzolino M, Brancaccio D. Transient decrease of serum bicarbonate levels with Sevelamer hydrochloride as the phosphate binder. Kidney Int. 2000;57(4):1776-1777.

48. Brezina B, Qunibi WY, Nolan CR. Acid loading during treatment with sevelamer hydrochloride: mechanisms and clinical implications. Kidney Int Suppl. 2004;90 Suppl:S39-S45.

49. Sonikian MA, Pani IT, Iliopoulos AN, Koutala KG, Marioli SI, Vlassopoulos DA. Metabolic acidosis aggravation and hyperkaliemia in hemodialysis patients treated by sevelamer hydrochloride. Ren Fail. 2005;27(2):143-147.

50. Marco MP, Muray S, Betriu A, Craver L, Belart M, Fernández E. Treatment with sevelamer decreases bicarbonate levels in hemodialysis patients. Nephron. 2002;92(2):499-500.

51. Grinfeld J, Inaba A, Hutchison AJ. Update and critical appraisal of sevelamer in the management of chronic renal failure. Open Access $J$ Urol. 2010;2:161-170.

52. Delmez J, Block G, Robertson J, et al. A randomized, double-blind, crossover design study of sevelamer hydrochloride and sevelamer carbonate in patients on hemodialysis. Clin Nephrol. 2007;68(6):386-391.

53. Harland C, Wrong O. Sevelamer 'carbonate': what's in a name? Nephrol Dial Transplant. 2010;25(4):1354-1355.

54. Ferreira A, Frazão JM, Monier-Faugere MC, et al; Sevelamer Study Group. Effects of sevelamer hydrochloride and calcium carbonate on renal osteodystrophy in hemodialysis patients. J Am Soc Nephrol. 2008;19(2):405-412.

55. Salusky IB, Goodman WG, Sahney S, et al. Sevelamer controls parathyroid hormone-induced bone disease as efficiently as calcium carbonate without increasing serum calcium levels during therapy with active vitamin D sterols. J Am Soc Nephrol. 2005;16(8):2501-2508.

56. Medi-Span ${ }^{\circledR}$ [homepage on the Internet]. Available from: http://www. medispan.com. Accessed February 25, 2014.

57. Manns B, Stevens L, Miskulin D, Owen WF Jr, Winkelmayer WC, Tonelli M. A systematic review of sevelamer in ESRD and an analysis of its potential economic impact in Canada and the United States. Kidney Int. 2004;66(3):1239-1247.

58. Manns B, Klarenbach S, Lee H, Culleton B, Shrive F, Tonelli M. Economic evaluation of sevelamer in patients with end-stage renal disease. Nephrol Dial Transplant. 2007;22(10):2867-2878.

59. St Peter WL, Fan Q, Weinhandl E, Liu J. Economic evaluation of sevelamer versus calcium-based phosphate binders in hemodialysis patients: a secondary analysis using centers for Medicare and Medicaid services data. Clin J Am Soc Nephrol. 2009;4(12):1954-1961.

60. Thompson M, Bartko-Winters S, Bernard L, Fenton A, Hutchison C, Di Iorio B. Economic evaluation of sevelamer for the treatment of hyperphosphatemia in chronic kidney patients not on dialysis in the United Kingdom. J Med Econ. 2013;16(6):744-755.

61. Bernard L, Mendelssohn D, Dunn E, Hutchison C, Grima DT. A modeled economic evaluation of sevelamer for treatment of hyperphosphatemia associated with chronic kidney disease among patients on dialysis in the United Kingdom. $J$ Med Econ. 2013;16(1):1-9.

62. Taylor MJ, Elgazzar HA, Chaplin S, Goldsmith D, Molony DA. An economic evaluation of sevelamer in patients new to dialysis. Curr Med Res Opin. 2008;24(2):601-608.

\section{Publish your work in this journal}

The International Journal of Nephrology and Renovascular Disease is an international, peer-reviewed open-access journal focusing on the pathophysiology of the kidney and vascular supply. Epidemiology, screening, diagnosis, and treatment interventions are covered as well as basic science, biochemical and immunological studies. The journal welcomes original research, clinical studies, reviews \& evaluations, expert opinion and commentary, case reports and extended reports. The manuscript management system is completely online and includes a very quick and fair peerreview system, which is all easy to use. Visit http://www.dovepress.com/ testimonials.php to read real quotes from published authors. 